\title{
Signature of the Spin Triplet Phase in $\mathrm{La}_{0.7} \mathrm{Sr}_{0.3} \mathrm{MnO}_{3} / \mathrm{Yba}_{2} \mathrm{Cu}_{3} \mathrm{O}_{7} / \mathrm{La}_{0.7} \mathrm{Sr}_{0.3} \mathrm{MnO}_{3}$ Trilayers
}

\author{
P. Przyslupski, K. Dybko, K. Werner-Malento, P. Aleshkevych, M. Wojcik \\ AND M. SAWICKI
}

Institute of Physics, Polish Academy of Sciences, al. Lotników 32/46, 02-668 Warsaw, Poland

\begin{abstract}
Differential dynamical subgap transport measurements were performed on LSMO/YBCO/LSMO trilayers to probe local evolution of the Andreev bound states which are manifested as the zero bias conductance peak. Dynamical conductivity $\mathrm{d} I / \mathrm{d} V$ vs. magnetic field measured in current in plane and current perpendicular to plane geometries show nonmonotonic behavior with maximum at about 500 Oe. The shape of the zero bias conductance peak measured in current in plane geometry is sharp, whereas zero bias conductance peak measured in current perpendicular to plane geometry demonstrates $\mathrm{V}$ shape. These shapes of the zero bias conductance peak were predicted theoretically for unconventional $p$-wave spin triplet phase superconductor and superconductor with $d$-wave symmetry of the order parameter, respectively.
\end{abstract}

PACS numbers: 74.25.Ha, 74.78.Fk, 75.70.Cn, 77.84.Bw

\section{Introduction}

An intriguing feature of the proximity effect between singlet superconductor (S) and nonhomogeneous ferromagnet $(\mathrm{F})$ is the possibility of generation of the triplet superconducting component [1]. Later it was theoretically predicted that the triplet component can also arise in the case of homogeneous ferromagnets but noncollinearly aligned in $\mathrm{F} / \mathrm{S} / \mathrm{F}$ structure. An $\mathrm{F} / \mathrm{S} / \mathrm{F}$ trilayer with homogeneous but noncollinear magnetization $[2,3]$ of the $\mathrm{F}$ layers is the simplest example of layered structure in which the triplet component could be generated. The triplet component (correlation between quasiparticles with parallel spins) arises as a result of the interplay between the Andreev reflection at the $\mathrm{S} / \mathrm{F}$ interfaces. The mechanism responsible for the generation of the triplet component is spin mixing and spin flip scattering event. The first process, represented by phase difference between waves of opposite spin orientation reflected from spin-active interface introduces triplet correlation at the superconducting side of the half metal/ HTSC boundary (HM/HTSC). In the second case these correlations are generated in the HM side [4]. Another theoretical approach [5] takes into account the spin dynamics. According to this model apart of the conversion between spin-singlet and spin triplet pairs an important role is also played by the formation of the composite state of a triplet pairs and magnon in the transport of the supercurrent in HTSC/HM heterostructures. There is a relatively good understanding [6] of the subgap transport in normal metal-high temperature superconductor contacts (N-HTSC) indicating the important role of the Andreev bound states (midgap states) which lead to the formation of zero bias conductance peak (ZBCP).

In this report we present the study of the subgap transport in $\mathrm{LSMO} / \mathrm{YBCO} / \mathrm{LSMO}$ trilayer in current in plane
(CIP) and current perpendicular to plane (CPP) geometry. Data show that low energy transport depends on crystallographic orientation of the trilayer with predominant V shape ZBCP measured in CPP orientation and sharp peak in CIP orientation. These data suggest that noncollinear alignment of magnetic moments in LSMO layers induces a spin triplet phase at YBCO and LSMO interface as indicated by the sharp peak measured in CIP geometry in accordance with the theoretical model.

\section{Experimental}

$\mathrm{LSMO} / \mathrm{YBCO} / \mathrm{LSMO}$ trilayer heterostructures were deposited by high pressure sputtering [7] from stoichiometric targets at $(100)\left[\left(\mathrm{LaAlO}_{3}\right)_{0.3}\left(\mathrm{Sr}_{2} \mathrm{TaAlO}_{6}\right)_{0.7}\right]$ (LSAT) substrates. After the deposition of the first LSMO layer, the sputtering chamber was opened and a part of the LSMO film was covered with another substrate. Then the consecutive YBCO and LSMO layer was deposited. Transport measurements were made using four point probe method. The step-like structure unables the measurements with CIP and CPP geometries. Dynamical conductance was measured using standard low frequency lock-in technique and Delta mode of the Keithley 6221 AC/DC programmable current source. The magnetoresistance was measured with current set to $10 \mu \mathrm{A}$. The magnetization of trilayers was measured using SQUID magnetometer.

\section{Results and discussion}

The differential conductance spectra $(\mathrm{d} I / \mathrm{d} V$ vs. $\left.V_{\text {bias }}\right)$ recorded in two geometries CIP and CPP for $\operatorname{LSMO}(22 \mathrm{~nm}) / \mathrm{YBCO}(20 \mathrm{~nm}) / \operatorname{LSMO}(10 \mathrm{~nm})$ trilayer are shown in Fig. 1 for comparison. Both spectra were 
recorded at the temperature $T=65.75 \mathrm{~K}$, slightly below the onset of the superconducting transition for this sample $\left(T_{\text {conset }}=75 \mathrm{~K}\right)$. The shape of the peaks differs qualitatively. The amplitude of the sharp peak in CIP geometry (solid line) increases twice within presented $V_{\text {bias }}$ range, whereas peak observed in CPP configuration is round and analogous change of its amplitude remains less than 10 percent (broken line). Both peaks naturally disappear above superconducting transition temperature.

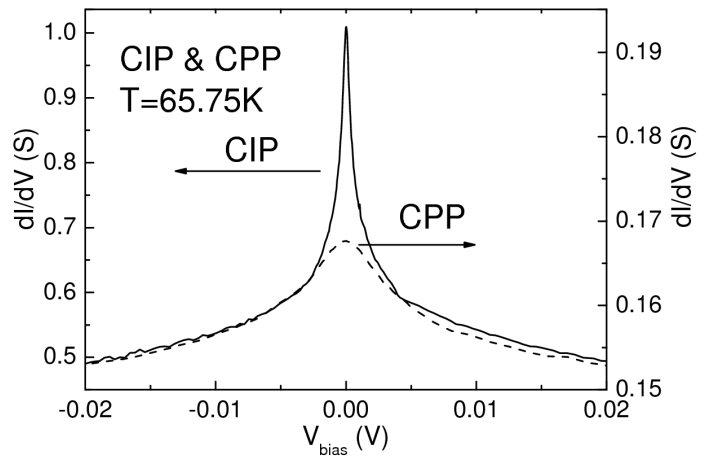

Fig. 1. Differential conductance vs. bias voltage recorded at zero magnetic field in the CIP and CPP geometries for $\operatorname{LSMO}(22 \mathrm{~nm}) / \operatorname{YBCO}(20 \mathrm{~nm}) /$ $\operatorname{LSMO}(10 \mathrm{~nm})$ trilayer.

In unconventional superconductors, contact spectroscopy reflects the internal phase of the pair potential and charge transport becomes phase sensitive. The appearance of $\mathrm{ZBCP}$ in the tunneling spectroscopy is due to the formation of mid-gap Andreev resonant states (MARS). The origin of MARS is due to the interference effect of quasiparticles at the interface, where injected and reflected quasiparticles are subjected to different sign of pair potential. The differences in the shape of the observed experimentally ZBCP in CIP and CPP configurations were predicted also theoretically [8] for $p$-wave and $d$-wave symmetry of the order parameter of the superconducting phase, respectively.

The evolution of the dynamical conductance spectra in magnetic field is presented in Fig. 2 and Fig. 3, respectively. The external magnetic field was applied in plane of the trilayer, in the direction perpendicular to the current flow in both configurations, CIP and CPP.

It is seen that the differential conductance increases with the increase of magnetic field, reaching maximum at about 500 Oe. The increase of conductance at the LSMO/YBCO boundary could be a signature of the long range proximity effect in the LSMO ferromagnet. The enhancement of proximity effect at this field could result from optimal domain configuration or optimal noncolinearity of top and bottom LSMO layers. At this field presumably the free energy of the triplet phase reaches maximum. Therefore the triplet pairs penetrate the manganite layers on relatively long distance. Theoretical model of the proximity effect in $d$-wave superconductor and ferromagnet with the domain walls structure predicts that

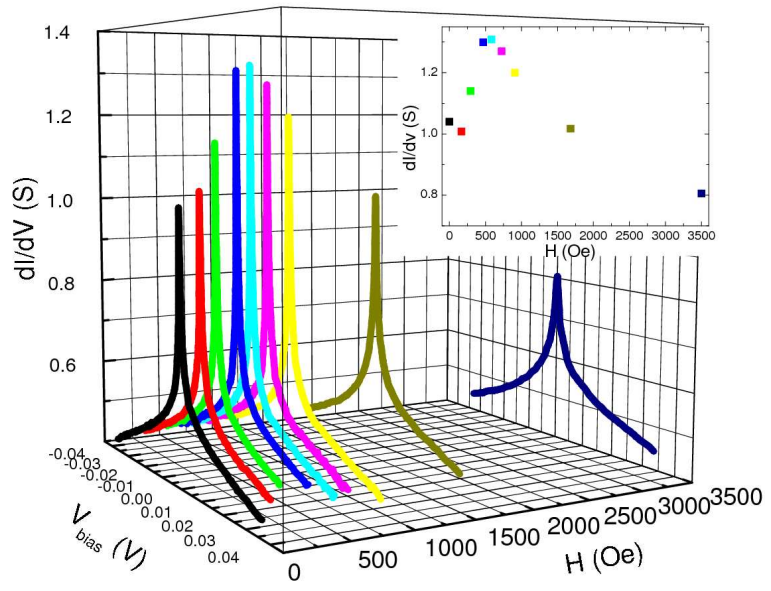

Fig. 2. Differential conductance of $\operatorname{LSMO}(22 \mathrm{~nm}) /$ $\mathrm{YBCO}(20 \mathrm{~nm}) / \operatorname{LSMO}(10 \mathrm{~nm})$ trilayer, $\mathrm{d} I / \mathrm{d} V$ vs. $V_{\text {bias }}$ and magnetic field measured in CIP geometry at $T=$ 65.75 K. Inset: maximum $\mathrm{d} I / \mathrm{d} V$ vs. $H$.

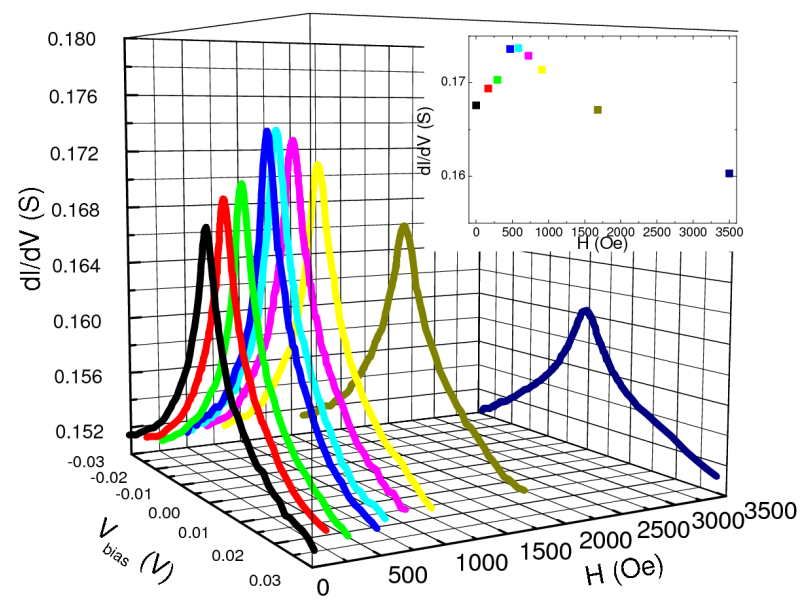

Fig. 3. Differential conductance of $\operatorname{LSMO}(22 \mathrm{~nm}) /$ $\mathrm{YBCO}(20 \mathrm{~nm}) / \operatorname{LSMO}(10 \mathrm{~nm})$ trilayer, $\mathrm{d} I / \mathrm{d} V$ vs. $V_{\text {bias }}$ and magnetic field measured in CPP geometry at $T=$ 65.75 K. Inset: maximum $\mathrm{d} I / \mathrm{d} V$ vs. $H$.

an inhomogeneous exchange field could enhance the proximity effect [9].

The magnetoresistance (Fig. 4) performed on similar trilayer $\operatorname{LSMO}(22 \mathrm{~nm}) / \mathrm{YBCO}(19 \mathrm{~nm}) / \operatorname{LSMO}(10 \mathrm{~nm})$ demonstrate a minimum of magnetoresistance (MR) at magnetic field of 500 Oe. The maximum of MR is observed for the coercive field both above and below the superconducting transition temperature. The minimum of $\mathrm{MR}$ is reached for magnetic field below the saturation of magnetic moment of the whole trilayer. We argue that this minimum is the result of the appearance of the spin triplet phase. Therefore, also the conductance shows the maximum of $\mathrm{d} I / \mathrm{d} V$ exactly for this field where the minimum of $\mathrm{MR}$ is observed. This means 


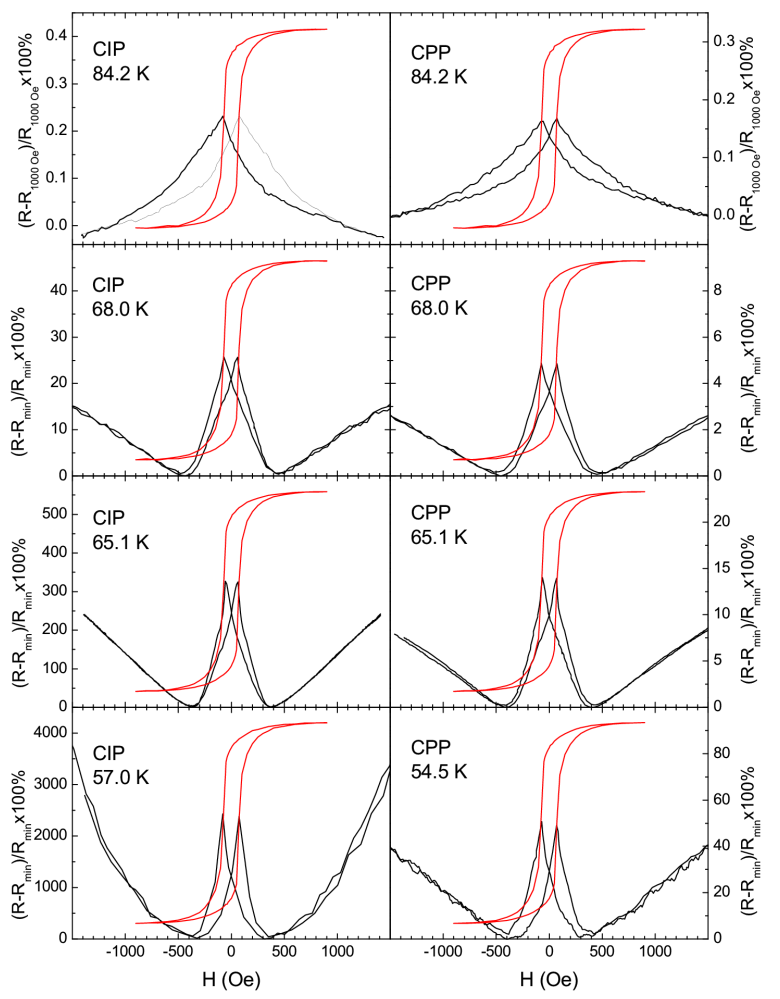

Fig. 4. Magnetoresistance obtained for $\operatorname{LSMO}(22 \mathrm{~nm}) / \mathrm{YBCO}(19 \mathrm{~nm}) / \operatorname{LSMO}(10 \mathrm{~nm})$ trilayer measured in CIP (left part) and CPP (right part) above and below superconducting transition $T_{\text {onset }}$. For clarity the $M(H)$ hysteresis loop is superimposed on $\operatorname{MR}(H)$ curves.

that at this field the magnetic domain structures of both bottom and top LSMO layers produce optimal magnetic noncollinearity necessary for the appearance of spin triplet phase.

The signature of the spin triplet phase was suggested based on neutron reflectometry measurements on YBCO/LCMO multilayers [10]. Similar results were obtained on another set of samples including the description of interface properties which play a significant role in the subgap transport [11]. The appearance of spin triplet phase was demonstrated also by means of microwave absorption measurements in LSMO/YBCO superlattices [12] indicating a strong frequency dependence in qualitative agreement with theoretical model [5].

In summary, the differential conductance measurements demonstrate a sharp peak indicating a $p$-wave symmetry of the order parameter of superconducting phase generated at LSMO/YBCO interface in agreement with theoretical predictions. The differential conductance measurements in CPP geometry demonstrate an existence of the Andreev bound states at the LSMO/ YBCO interface where the $\mathrm{V}$ shape of ZBCP was observed. The $\mathrm{V}$ shape of the $\mathrm{ZBCP}$ corresponds to the $d$-wave symmetry of the order parameter. The difference in the shape measured in CPP and CIP geometry is a result of large influence of magnetic inhomogeneity of magnetic field in CIP geometry and its much smaller impact in CPP geometry.

\section{Acknowledgments}

This work was supported by the Ministry of Sciences and Higher Education (MNiSW) under research project for the years 2010-2014(N N202 204338).

\section{References}

[1] F.S. Bergeret, Phys. Rev. Lett. 86, 4096 (2003).

[2] K. Halterman, P.H. Barisic, O.T. Vallas, Phys. Rev. Lett. 99, 127002 (2007).

[3] Z. Ping, D.Y. Xing, Phys. Rev. Lett. 98, 057005 (2007).

[4] M. Eschrig, J. Kopu, J.C. Cuveas, G. Schon, Phys. Rev. Lett. 90, 137003 (2003).

[5] S. Takahashi, S. Hikino, M. Mori, J. Martinek, S. Maekawa, Phys. Rev. Lett. 299, 1719 (2007).

[6] S. Kashiwaya, Y. Tanaka, Rep. Prog. Phys. 63, 1641 (2000).

[7] P. Przyslupski, I. Komissarov, W. Paszkowicz, P. Dluzewski, R. Minikayev, M. Sawicki, Phys. Rev. B 69, 134428 (2004).

[8] Y. Tanaka, S. Kashiwaya, Phys. Rev. B 70, 012507 (2004).

[9] A.F. Volkov, K.B. Efetov, Phys. Rev. Lett. 102, 077002 (2009).

[10] J. Hopler, J. Sthan, CH. Nidermayer, V.K. Malik, H. Boyanfif, A.J. Drew, M. Rossle, A. Buzdin, G. Cristiani, H.U. Habermeier, B. Keimer, C. Bernhard, Nature Mater. 8, 315 (2009).

[11] K. Dybko, K. Werner Malento, P. Aleshkevych, M. Wojcik, M. Sawicki, P. Przyslupski, Phys. Rev. B 80, 144504 (2009).

[12] P. Gierlowski, K. Werner Malento, P. Przyslupski, C.J. van der Beek, Appl. Phys. Lett. 95, 172511 (2009). 